Cahiers de recherche sociologique

RECHERCHE

SOCIOLOGIQUE

\title{
Les sculptures pop (1966-1969) de Claire Hogenkamp : art critique ou mannequins de pacotille?
}

\section{Rose-Marie Arbour}

Numéro 16, printemps 1991

Art, artistes et société

URI : https://id.erudit.org/iderudit/1002128ar

DOI : https://doi.org/10.7202/1002128ar

Aller au sommaire du numéro

\section{Éditeur(s)}

Département de sociologie - Université du Québec à Montréal

ISSN

0831-1048 (imprimé)

1923-5771 (numérique)

Découvrir la revue

Citer cet article

Arbour, R.-M. (1991). Les sculptures pop (1966-1969) de Claire Hogenkamp : art critique ou mannequins de pacotille? Cahiers de recherche sociologique, (16),

55-73. https://doi.org/10.7202/1002128ar
Résumé de l'article

$\mathrm{Au}$ départ l'auteure souligne que les travaux de la sculpteure qu'elle étudie font partie du courant figuratif ayant contesté, au cours des années soixante, le formalisme en arts visuels. En commentant des oeuvres présentées à

l'exposition « People in the Park » l'auteure montre que Claire Hogenkamp a pratiqué une satire marquée, mais indulgente (non culpabilisante) de la féminité et de la masculinité construites essentiellement sur des rapports de séduction hypercodés suggérés par l'imagerie publicitaire et la photographie commerciale. Elle précise que le contenu de ces oeuvres, par leur effet de distanciation du réel, montre l'évidence du règne du faux, particulièrement dans le cas de la féminité construite par les médias commerciaux. Finalement, l'auteure démontre que cette recherche d'Hogenkamp constitue une critique sociale forte des comportements homme-femme stéréotypés, ce qui du coup révèle la fonction idéologique de l'art, c'est-à-dire sa nécessaire portée critique. 


\section{Les sculptures pop (1966-1969) de Claire Hogenkamp: art critique ou mannequins de pacotille?}

Rose-Marie ARBOUR

À l'été 1969, une exposition intitulée People in the Park: Figurative Sculpture of the Sixties présentait des sculptures en plein air sur le site du Reservoir Park (Ontario) ${ }^{1}$. La figuration en était le dénominateur commun, ce qui était remarquable dans un contexte artistique dominé par les approches optiques et formalistes - l'exemple montréalais étaient les Plasticiens (Guido Molinari, Claude Tousignant...) - et dans une période particulièrement intéressée aux nouvelles technologies (matériaux, structures, concepts, processus), particulièrement en sculpture.

Il convient de rappeler l'importance, sur les plans conceptuel, idéologique et esthétique, des thèses modernistes qui avaient alimenté les transformations de l'art abstrait depuis les années cinquante aux États-Unis - plus particulièrement celles de Clement Greenberg, principal théoricien de l'autoréférentialité (et par là de l'abstraction). Greenberg insistait sur la spécificité des disciplines (la "pure opticality" en peinture) et leur autonomie - chaque art se développant dans un processus d'autocritique qui l'amenait à se retrancher dans son strict domaine de compétence. Cette théorie marqua profondément la peinture américaine des années cinquante et soixante: "L'essence du modernisme, à mon avis, c'est d'utiliser les méthodes propres d'une discipline pour critiquer cette même discipline ${ }^{2}$..."

Le formalisme en arts visuels se définissait alors en opposition à toute forme figurative, à toute forme de réalisme, à tout contenu: "Le contenu doit être dissout si complètement dans la forme que l'œuvre d'art ou de littérature ne puisse être

1 L'exposition était organisée par la Rothman's Gallery, Stratford, Ontario.

2 C. Greenberg, "Modernist Painting", in Art and Literature, no 4, printemps 1965. Traduit dans Peinture, Cahiers théoriques, nos 8-9, février 1974. 
réduite en partie ou en tout à quoi que ce soit d'autre que soi (...) le sujet ou contenu devient quelque chose à éviter comme la peste" ${ }^{\text {. }}$.

Aussi la résurgence du figuratif en art apparut-elle à certains artistes et critiques formalistes, comme une hérésie. La figuration en art s'était manifestée à la fin des années cinquante dans le courant pré-pop aux États-Unis, avec Rauschenberg par exemple, et, un peu plus tard au Québec, dans les œuvres peintes d'une Kittie Bruneau ou les gravures d'un Dumouchel ou d'un Pierre Ayot ${ }^{4}$.

Les jeunes artistes liés de près ou de loin à ce nouveau courant figuratif, s'opposaient nommément à l'expressionnisme abstrait et à l'abstraction lyrique en peinture, contestant la valeur de vérité et d'authenticité dont une gestualité instinctive et une organisation intuitive de la toile s'étaient voulues porteuses. La peinture gestuelle était portée par une conception de l'artiste comme shaman ou héros, ce qui n'allait décidément plus dans le sens des jeunes artistes, beaucoup plus critiques face à leur rôle d'artistes dans la société. En Europe et en Amérique du Nord des courants figuratifs démentaient ainsi une croyance proprement moderniste selon laquelle le progrès en art va vers une plus grande abstraction par une plus grande réduction de ses moyens matériels, formels et visuels. La jeune génération d'artistes montréalais s'opposa également à l'art exclusivement géométrique (comme celui des Plasticiens) et dont les prémisses formalistes coupaient toute référence à la réalité extérieure.

Dans un tel contexte, l'exposition People in the Park confirmait, en sculpture, qu'il y avait une opposition majeure au formalisme moderniste et que l'art n'était pas considéré par les exposants comme une pratique aseptisée, coupée du monde extérieur.

Leurs œuvres critiquaient sur le mode de l'humour un certain mythe de la technologie en art qui avait un temps ajouté une notion fonctionnaliste à la "pure opticality". A une exposition à la Galerie du Siècle (Montréal, 1967), La Nourrice de Serge Cournoyer avait nargué la haute technologie en faisant référence à une fonction première d'alimentation chez le nouveau-né: la sculpture "organique" selon l'artiste, transformait l'humidité de l'air en gouttes d'eau qui, tombant sur une petite plante à intervalles fixes, scientifiquement réglés sur les besoins de la plante, la nourrissaient.

3 C. Greenberg, "Avant-garde and Kitsch", Art and Culture, Beacon Press, 1966. p. 3-21.

4 Elle n'avait cessé d'exister chez des artistes aussi différents les uns des autres que Jean-Paul Lemieux, Jean Dallaire et Alfred Pellan. 
Claire Hogenkamp ${ }^{5}$, qui faisait partie de cette exposition d'un groupe de jeunes sculpteurs canadiens, était de cette nouvelle génération d'artistes qui, à Montréal, comprenait Serge Lemoyne, Jean Noël, Jean-Marie Delavalle, Reynald Connoly, Jean-Claude Lajeunie, Irène Chiasson, Marc Lepage, Maurice Demers, Pierre Ayot, Richard Lacroix ... La polyvalence des moyens techniques et des technologies, et l'affirmation du contenu par la figuration-narration contribuaient à alimenter un courant politique de critique sociale.

Je propose ici une lecture des œuvres (1966-1969) de Claire Hogenkamp, une des rares femmes à faire partie de ce nouveau courant figuratif critique. Cette lecture se fera tant sur le plan des éléments plastiques et du dispositif scénique que sur le plan du contenu. Attirer ici l'attention sur le fait que cette artiste est une femme vise à rappeler qu'elle venait après une décennie où les femmes artistes avaient été les leaders de la peinture post-automatiste (lyrique et abstraite) qui a dominé la scène de l'art vivant (ou de l'art dit avancé) au cours des années 1955-1965 au Québec et au Canada. Après 1965, leur nombre avait considérablement diminué. Mais la question qui nous occupe est de savoir ou du moins d'essayer de préciser comment Hogenkamp s'est inscrite dans un courant majoritairement représenté et identifié à des hommes et si elle l'a marqué d'une quelconque manière. Sans faire appel à une spécificité stylistique féminine pour analyser son apport ${ }^{6}$, le genre sera considéré ici comme un des éléments ayant pu entraîner un certain contenu social et politique dans les sculptures de Claire Hogenkamp.

\section{Les relations homme-femme}

La conservatrice de l'exposition People in the Park, Dorothy Cameron, avait bien résumé l'enjeu politique de l'art actuel qu'elle y présentait, en dichotomisant la scène artistique canadienne selon deux vecteurs principaux:

People in the Park constitue une suggestion: à savoir que la forme humaine, source la plus ancienne et la plus exploitée de notre connaissance des formes, est loin d'être une impasse en sculpture contemporaine (malgré les multiples annonces de sa mort prématurée) (...), que les dimensions formalistes et humanistes de l'art qui ont co-existé et échangé pendant des milliers d'années continuent de le faire aujourd'hui selon l'adaptation et l'exploitation par des artistes figuratifs des innovations techniques et matérielles de l'avant-grarde formaliste, (...) quand cette dernière ne se préoccupe que de démonter les éléments de l'environnement de l'homme, il y en a d'autres qui continuent à avoir un intérêt primordial pour l'être humain lui-même comme faiseur et

5 C. Hogenkamp est née à La Haye (Hollande) en 1940. Arrivée au Canada en 1946, elle a vécu à Montréal depuis 1947. Sa carrière artistique en ce pays s'est terminée au début des années soixante-dix.

6 C'est ce qu'avaient fait les critiques d'art pour la peinture gestuelle lyrique postautomatiste pratiquée par les femmes artistes de la génération précédente. 
miroir psychique de l'environnement (...), que cette petite mais tenace minorité qui donne actuellement une vue renouvelée de l'expression figurative pourrait bien répondre à un besoin général de l'esprit contemporain que le minimalisme a mis au régime pendant trop longtemps?

Dans cette exposition, Claire Hogenkamp présentait ses baigneurs (Sunworshippers) sur un radeau amarré sur l'étang formé par le réservoir, dans le parc où se situait l'ensemble des œuvres exposées. L'effet saisissant de vraisemblance a justifié le nom de "post-pop" que lui a donné la critique ${ }^{8}$. Le matériau utilisé était de la résine de polyester moulée, polychromée avec de la peinture époxy ${ }^{9}$. L'artiste avait utilisé de vrais verres fumés pour le Male Sunworshipper, de vrais faux-cils et une queue de cheval en dynel pour sa compagne, un vrai bonnet de bain pour une autre baigneuse... Cette mise en scène de personnages adonnés à une activité qui métaphorisait la société de loisir et de consommation (la valeur indicielle et symbolique d'un bain de soleil et du bronzage), s'intégrait admirablement bien au site. Par l'utilisation des lieux réels non seulement comme cadre mais comme espace structurant la sculpture ellemême, Claire Hogenkamp était plus proche d'un Oldenburg que d'un Warhol (tous deux emblématiques du Pop Art américain), plus proche également d'une Niki de Saint-Phalle et d'un Segal pour le traitement matériel de ses personnages et des thèmes.

Hogenkamp s'était déjà révélée dans des œuvres antérieures une satiriste feutrée, discrète. Sa stratégie consistait à mettre en scène des personnages ordinaires, tirés de la vie quotidienne dans des postures qui pouvaient s'approcher d'une caricature "douce". Sa satire n'avait rien de grinçant: c'était plutôt un constat de réalités familières dont la sienne propre. Dans l'exposition de Stratford, l'une des baigneuses était un autoportrait.

7 D. Cameron, "People in the Park", Art and Artists, novembre 1969, p. 16-17. Traduction de l'auteur.

8 D. Cameron, "New Sculptures at Rothman's Gallery, Stratford", Artscanada, août 1969, p. 34-35.

9 Le polyester coloré utilisé était une matière courante dans la fabrication des objets commerciaux. Dans les années soixante, il n'était pas encore considéré comme matériau artistique. Voici la description du procédé d'utilisation du polyester faite par un artiste qui en faisait l'expérimentation: "Cette matière peut s'appliquer en couches successives, épaissies par des charges de silice et renforcées de fibre de verre jusqu'à l'obtention du volume désiré. La sculpture est ensuite peinte avec des couleurs acryliques qui adhèrent parfaitement au support. Cette résine sera sans doute capable d'attirer d'autres artistes pour des raisons différentes" (C. Blin, "L'artiste et les matières plastiques", Culture Vivante, no 13, mai 1969, p. 24. 


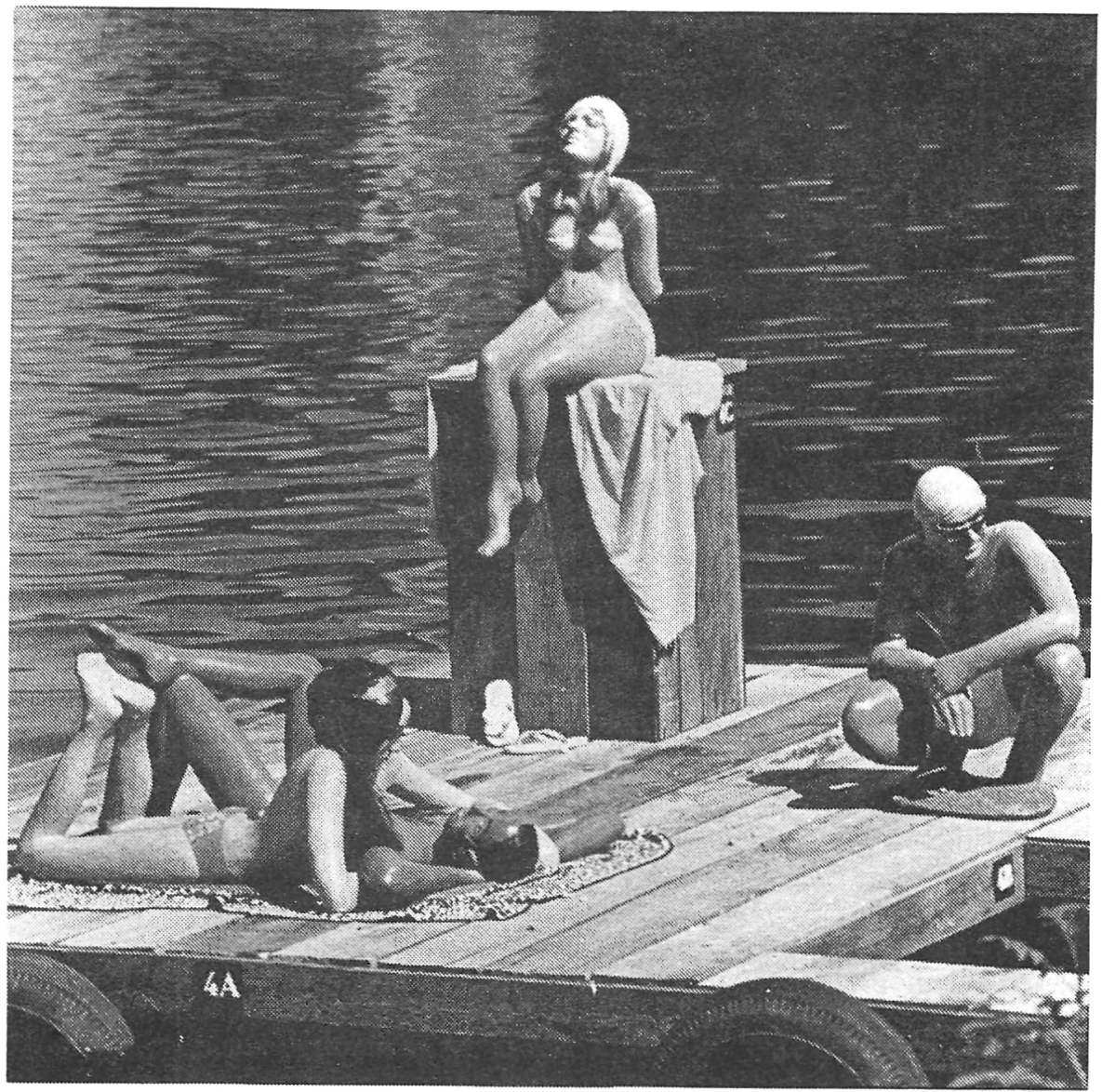

Claire Hogenkamp, Reclining Sunworshippers, Polyester, peinture epoxy (personnages grandeur nature), 1969. 
Sa compréhension des rapports homme-femme s'est construite au fil des années (depuis le début des années soixante) autour de thèmes et de sujets sociaux abordés et traités dans des œuvres aux techniques diverses (gravure, collage, dessin, sculpture). Ces thèmes avaient touché au féminisme, au logement, à la mode vestimentaire, à la mariée, au héros, au travail ouvrier, aux loisirs de masse.

Dans les œuvres de cette exposition en plein air, la satire était marquée mais indulgente: la surface rutilante et colorée de l'american way of life, les gestes schématisés et suggestifs des personnages (hommes et femmes), les maquillages appuyés à la façon des annonces publicitaires - tout concordait à représenter ces "êtres de loisirs" de manière distanciée et critique sans pour autant se charger d'une quelconque culpabilité. Cette représentation révélait une conception de la féminité et de la masculinité construite essentiellement autour de la séduction. En incluant un personnage-autoportrait dans la scène, l'artiste adoptait une position qui n'en était pas une de maîtrise par rapport aux scènes et personnages décrits; elle était à la fois objet et sujet. Cette ambivalence sera, une décennie plus tard, revendiquée puis théorisée par les artistes et théoriciennes féministes.

Les tableaux vivants d'Hogenkamp n'avaient rien d'une dénonciation du corps féminin pris comme objet utilitaire sexuel à la manière de Ann James. Critique féroce de la réduction des femmes à des "meubles sexuels", la sculpture de James, en céramique, avait la forme d'une femme-chaise: What's a Nice Girl Like You Doing in a Place Like this? (1967) faisait partie de la même exposition. Claire Hogenkamp était plutôt complice de ses propres personnages; elle ne les érigeait pas en concentrés accusatoires de la difficulté de vivre ou de communiquer. Il n'y avait pas non plus chez elle cette dimension exploratoire sur le plan formel et virtuel qu'on retrouve chez Michael Snow (Sideway). Depuis 1962, Snow projetait une silhouette profilée de femme marchant en plans diversement orientés: cette silhouette découpée constituait une forme paradigme de son travail artistique qu'il reprenait sous divers modes (photo, film, vidéo). Justement, chez Snow, il n'y avait rien de l'image critique, la femme qu'il découpait inlassablement était plutôt un objet de consommation type présente dans l'esprit de constat comme le faisaient plusieurs artistes du pop art.

Chez Hogenkamp, la figure humaine, féminine ou masculine, n'était pas une forme anonyme et le schématisme des corps (que d'aucuns virent comme un emprunt littéral aux mannequins des vitrines de grands magasins) était contrebalancé par des accessoires réalistes, des surfaces brillantes et très colorées, modelées à la main comme l'auraient été des formes en papier mâché, ce qui donnait à la texture un aspect irrégulier, non fini ("fait main"). 


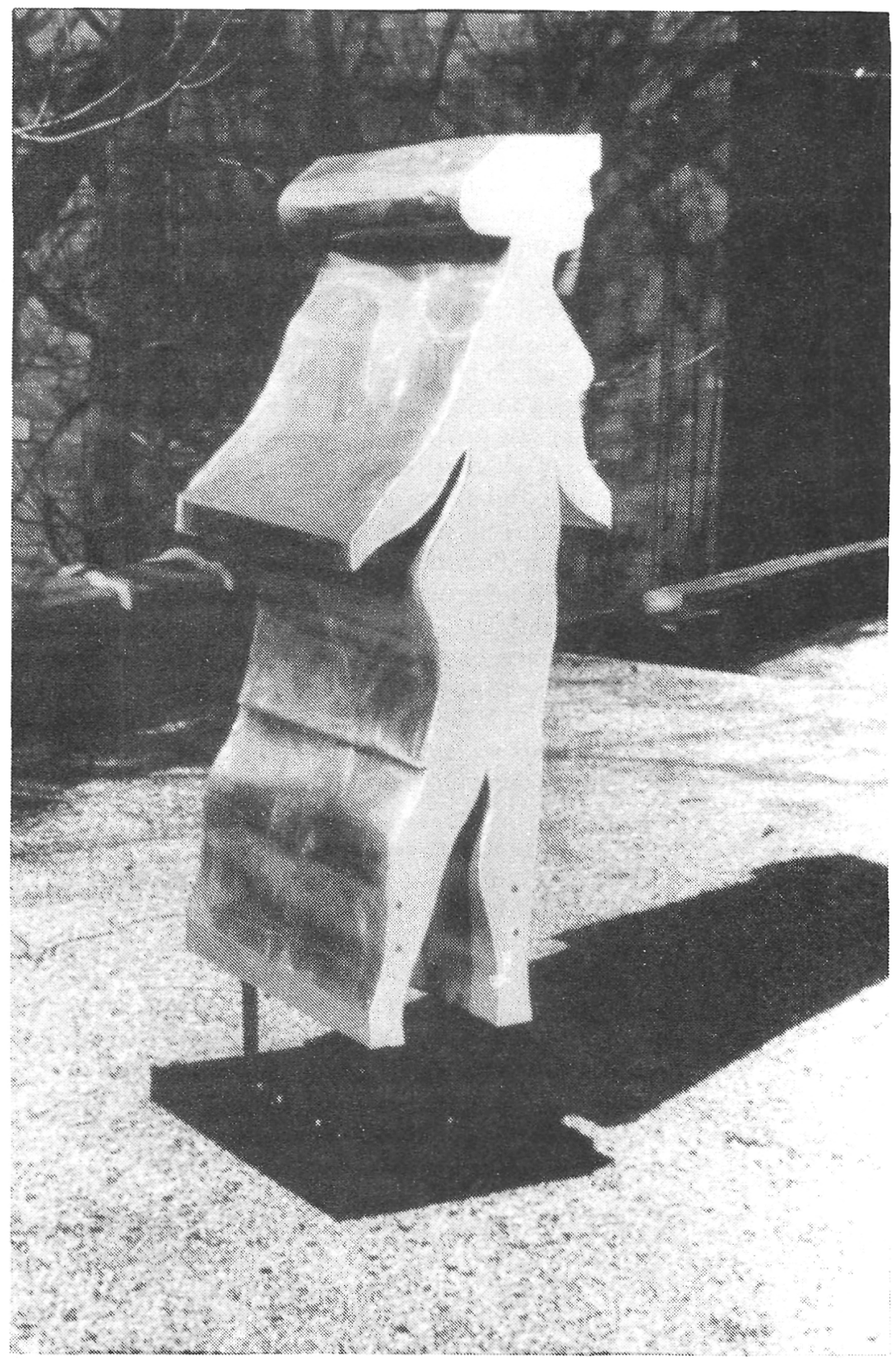

Michael Snow, Sydeway, aluminium peint, base en bois, hauteur 1,78m, 1962. 
Claire Hogenkamp visait à représenter ce qui était par ailleurs si difficile à cerner dans les rapports entre hommes et femmes en mettant en scène un état d'être plutôt que des formes réalistes, en mettant en scène ce moment volatile du plaisir de la séduction - moment de suspension dans le temps et l'espace, là où la sensation d'une rencontre (amoureuse) s'amorce. C'était ainsi un certain discours érotique du corps et de ses relations à l'espace et à d'autres corps qui était formalisé dans ces figures hyper codées, disposées dans des postures et gestes stéréotypés et banals, mais les figures étaient imprégnées de sensations singulières qui les posaient comme "êtres sentant", différents ainsi des mannequins de commerce tout en ayant été réalisés selon les mêmes codes formels et avec les mêmes matériaux.

Au milieu des années soixante, la portée critique de la "nouvelle figuration" en art avait suscité, chez le critique d'art américain Harold Rosenberg, une affirmation fort discutée: "The position of the artist as a critic of society is the essential foundation for the continuation of art", avait-il déclaré. Vingt ans plus tard, le débat était toujours ouvert et la critique d'art américaine, Lucy Lippard, affirmait au début des années quatre-vingts et dans un sens plus radical encore, que le seul art d'avantgarde était au Chili, sous le régime Pinochet, sous forme d'arpilleras ${ }^{10}$ réalisés par les femmes, justement en ce qu'ils véhiculaient des messages de résistance face au régime d'oppression sociale des militaires. Selon ses termes l'avant-garde artistique était vue comme devant assumer un rôle d'opposition politique aux forces d'oppression et une fonction militante au sein de la société, comme l'avaient fait les avant-gardes artistiques du début du XXe siècle (les Russes particulièrement). Elle refusait d'identifier l'avant-garde aux seules œuvres exploratoires sur les plans formel et visuel, comme l'avait prôné le courant formaliste dominant aux Etats-Unis depuis la fin des années cinquante.

Au Québec, Claire Hogenkamp et les artistes de sa génération refusaient l'hégémonie de l'abstraction gestuelle expressive en peinture mais aussi la valorisation exclusive de la pure opticalité de la peinture des Plasticiens. Ils adoptaient non seulement la figuration mais aussi des modes éclatés d'intervention dans le milieu de l'art (happenings), s'appropriaient des objets comiques et opposés au "bon goût" et plusieurs d'entre eux assumaient et réaffirmaient une fonction critique en art. La multidisciplinarité et la polyvalence des matériaux et des techniques conduisaient certains à faire des "actions" et des mises en situation qui avaient toutes plus ou moins pour objectif de rapprocher l'art dit avancé du "grand" public.

À propos de l'exposition présentée à la Galerie du Siècle, Sept sculpteurs du Québec, où Claire Hogenkamp était la seule femme ${ }^{11}$, le critique d'art canadien Barry Lord rappela, deux ans plus tard, l'existence d'un "nouveau réalisme" ou d'un

10 Sortes de couvertures cousues et brodées à la main.

11 Exposition de huit sculpteurs à la galerie du Siècle, décembre 1967-janvier 1968: Jacques Cleary, Serge Cournoyer, Jean-Marie Delavalle, Claire Hogenkamp, JeanClaude Lajeunie, Jean Noël, Serge Otis, Roger Paquin. 
"super-réalisme" post-pop au Canada, dont la première manifestation était justement l'exposition People in the Park où les personnages de Claire Hogenkamp l'avaient particulièrement impressionné ${ }^{12}$.

À cette occasion, un manifeste du réalisme perceptuel avait été publié en 1969 par Jack Chambers ${ }^{13}$, qui n'avait pas été étranger au courant qui se dessinait en faveur d'une représentation figurative réaliste au Canada anglais. Dans ce manifeste, l'emphase était mise sur les propriétés matérielles de la peinture comme entreprise "déconstructive" et l'importance de la photographie comme matériau actuel pour les peintres.

\section{L'instantané photographique comme modèle}

La photographie permet d'éclairer certains aspects des œuvres de Claire Hogenkamp. L'emprunt à ce médium peut être détecté dans la disposition psychologique des personnages les uns par rapport aux autres - disposition qui reproduit l'effet d'instantané propre à la photographie. Dans chacun des groupes la plupart, des couples - les personnages sont en arrêt, figés dans des attitudes stéréotypées; le temps est comme suspendu. Ce qui est ici particulier, c'est le renversement des termes qui régissent habituellement le rapport de séduction: c'est la femme, et non l'homme, qui joue le rôle actif, qui adopte les positions physiques et les rôles traditionnels de l'homme (Sunworshippers) ${ }^{14}$. Mais le couple en question ici tout comme les couples de danseurs ${ }^{15}$, n'en reste pas moins prisonnier d'une sorte de cube virtuel.

Les images médiatisées de l'amour - dont les romans Harlequin sont si friands - surdéterminent la conduite sociale des personnages: les codes des corps sont ceux de la publicité et de la photographie commerciale. Calqués sur ceux des médias, ils sont aussi et en même temps le produit d'un ensemble technologique et socioculturel plus vaste qu'ils mettent en scène par ricochet, le tout emblématisé par le thème de la séduction. En fait ces codes sont ceux de la société de consommation (des signes), devenus à la fois le sujet des œuvres d'Hogenkamp et aussi l'objet qui structure les représentations.

12 "The most profound and passionate of the works are Claire Hogenkamp's two pairs fibreglass dancers, Discotheque Couple and Waltzing Couple." B. Lord, "The Eleven o'clock News in Colour. Realism(e)s, realists, tableaux-vivants, painting, photography photo-sculpture and slide-shows: documentation" Artscanada, vol. XXVII, no 3, juin 1970, p. 4-5.

13 J. Chambers, Artscanada, octobre 1969.

14 Cette même position, radicalisée, se trouve dans le couple de mariés de la Chambre nuptiale de Francine Larivée (1976).

15 Discotheque Couple, 69 1/2" et Waltzing Couple 69 en résine polyester polychrome. 
Au moment où l'effervescence extraordinaire que l'Expo 67 avait suscitée, aux plans technologique, social et culturel, était retombée, l'exposition présentée à la Galerie du Siècle apparaissait à certains critiques comme un "souffle nouveau" sur la sculpture. On précise cette "nouveauté" en faisant l'inventaire de ses procédés et de ses stratégies: rejet des matériaux traditionnels (métal, bois, pierre), rejet de l'art moderniste (post-automatisme pictural, plasticisme), traitement anonyme des matériaux et des formes: "Les sculpteurs créent des objets propres, à l'aspect aussi impersonnel que s'ils sortaient d'une usine ${ }^{16}$." Un rapport visuel et formel était établi entre sculptures et environnement technologisé (machines, objets manufacturés) et on notait le retour d'une "nouvelle figuration": Serge Otis, JeanClaude Lajeunie, Claire Hogenkamp. Le court bilan permettait d'établir un certain lien entre objet d'art et contexte socio-culturel et technologique: malgré les moyens propres à chacun de ces artistes, les critiques soulignèrent leur filiation avec Dada, mais aussi le désir de dérision et de contestation des valeurs liées à l'univers de consommation. Ces sculpteurs ne tentaient surtout pas de combler le fossé entre la technologie et l'art; avec leurs machines qui étaient des anti-machines et leurs mannequins qui étaient des anti-mannequins, ils tentaient de les renvoyer dos à dos. Cette critique de la technologie par l'art, plus ou moins assumée selon les artistes, soulignait la distance entre les sculptures et la réalité qu'elles [dé]construisaient. Chez Claire Hogenkamp: le traitement "organique" (traces de la main de l'artiste) propre aux matériaux artistiques traditionnels (bois, métal) était ici ironiquement repris dans le traitement d'un matériau synthétique (résine de polyester) industriel et anonyme et dans le traitement des personnages médiatiques. Les traces de la main de l'artiste, qui avaient traditionnellement signalé sa présence derrière l'œuvre, étaient ici présentes comme autant de marques de son absence - les personnages apparaissaient comme produits par les codes sociaux de comportement plutôt que créés par l'artiste, individu singulier et unique.

Dans ses sculptures, la méthode d'Hogenkamp consistait à faire se côtoyer techniques de masse et techniques artisanales: les surfaces des corps lisses et simplifiées, brillantes et colorées, évoquent les matériaux synthétiques eux-mêmes comme signes de la société industrielle avancée. Mais contrairement à un Duane Hanson (sculpteur américain hyperréaliste), les corps ne sont pas modelés d'une façon illusionniste et naturaliste de sorte qu'il n'est pas possible d'y croire; l'illusionnisme ne joue pas son plein effet. Nous sommes en présence de "mannequins" certes, mais leur fonction est d'évoquer et de parodier une situation, un comportement, plutôt que d'en offrir l'illusion. Les accessoires réels ajoutés ont pour fonction de souligner le réalisme de l'évocation, de la rendre quasi-grotesque en rappelant l'importance des objets dans notre société, objets qui sont aussi des signes (objets de communication): lunettes fumées, chemisier, perruque, bonnet de bain. Les vêtements sont par ailleurs schématisés comme l'est l'anatomie des personnages, sans nuance ni subtilité. Ces vêtements ont pour fonction de situer

16 L. Lamy, "Sculpture d'aujourd'hui: fleurs, espaces, fusées", Le Devoir, 16 décembre 1967. 
historiquement, sexuellement et socialement les personnages, de montrer leur classe sociale, leur travail ou leurs loisirs, de les situer dans l'espace culturel (plage, discothèque).

\section{L'effet comme sens}

Les groupes sculptés (les Baigneurs, Sun Worshippers) ont un air factice malgré de "vrais" accessoires (perruques, lunettes soleil, bonnet de bain) qui ont pour effet d'accentuer encore davantage encore le simulacre dans la représentation des émotions et situations sentimentales qu'incarnent les couples.

Le contenu de ces œuvres est justement là, dans la rencontre paradoxale d'une iconographie stéréotypée et "commerciale" et de sentiments et attitudes supposés être réels, en ceci qu'ils relèvent des rapports interpersonnels amoureux. À la différence d'une scène d'un roman Harlequin, la mise en scène des personnages ne produit pas l'illusion de vérité mais, par un effet de distanciation, l'évidence du règne du faux. Le sentimentalisme plastifié se révèle comme produit instantané du vide marquant les relations interpersonnelles des consommateurs et consommatrices de signes (attitudes liées au genre sexuel dans le loisir et la séduction).

\section{Une esthétique de l'instantanéité des rapports de couple}

Paradoxalement, on a reproché à Hogenkamp d'esthétiser la banalité quotidienne ${ }^{17}$. À propos de l'ensemble des œuvres présentées dans l'exposition, Yves Robillard aurait voulu voir pousser plus avant le rôle social de l'artiste dans l'espace culturel et social et, plutôt que de faire de nouveaux objets en sculpture, il l'appelait à s'engager dans l'environnement urbain et à délaisser les objets sculptures. Ces objets rivalisaient, selon lui, avec les éléments de la vie quotidienne qu'il fallait au contraire valoriser et il entrevoyait la participation du public dans des espaces "qui modifient vraiment son sens de la réalité, sa façon d'être dans cette réalité et qu'il ne puisse pas y échapper".

\section{Une technologie ironisée}

Pour mieux saisir la portée de l'exposition de sculptures à la Galerie du Siècle, il faut revenir sur l'importance qu'avaient la haute technologie et les machines dans l'imaginaire public. Les jeunes artistes refusaient d'intérioriser ce fonctionnement technologique, conscients du danger d'homogénéiser les rapports entre les individus.

17 Y. Robillard, "Nouvel assaut contre les objets d'art", La Presse, 9 décembre 1967. 


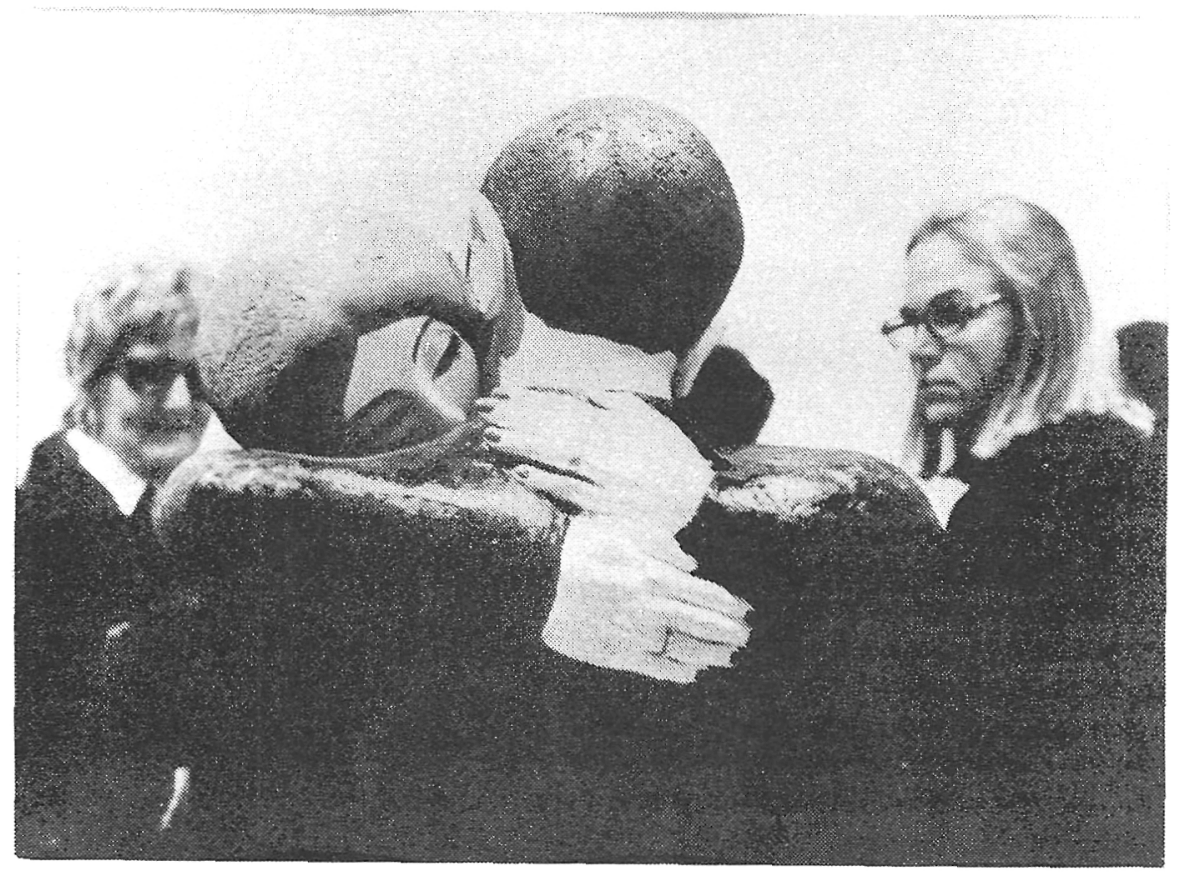

Claire Hogenkamp, Couple de discothèque, polyester, peinture epoxy, hauteur 2,10m, 1969-70. 


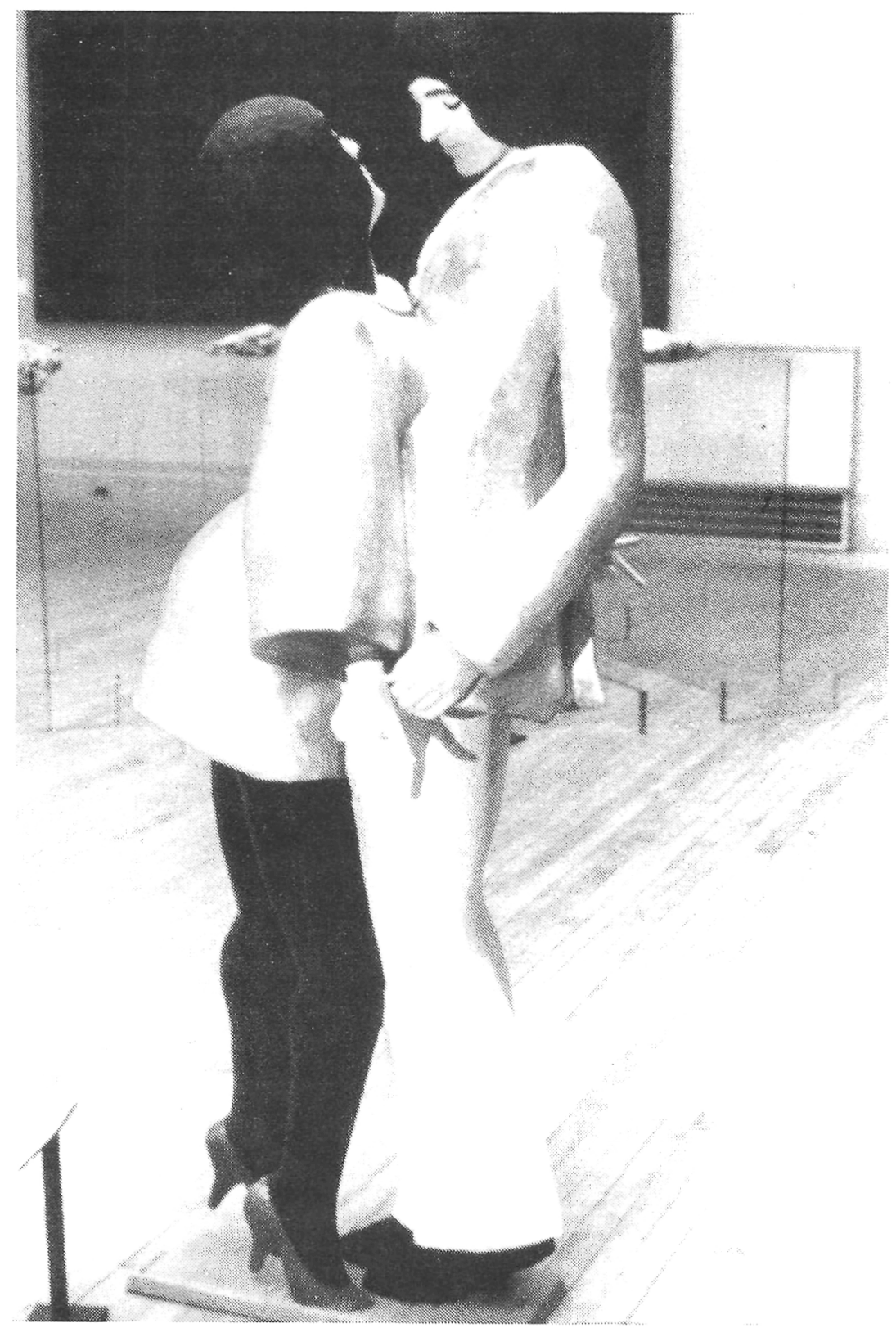

Claire Hogenkamp, Discotheque Couple (détail avec spectatrices), peinture epoxy, 1969-70. 
C'est à propos des œuvres de cette exposition qu'on parla d'anti-machine ${ }^{18}$, pour bien marquer la distance entre le regard des artistes et la technologie d'une société présumément juste. Les personnages d'Hogenkamp, étaient des anti-mannequins. Ses personnages relèvent d'une imagerie commerciale et populaire. S'ils empruntaient des traits aux mannequins (matériaux, postures, anonymat des traits), par renversement du discours, ils disaient autre chose que la pure consommation de signes et d'objets. L'accès de plain-pied du public aux sculptures posées à même le sol sauf exception (à l'exposition de Stratford (1969) où elles étaient installées dans un bassin d'eau ${ }^{19}$ ), manifestait une conception non hiérarchisée de l'art et son ouverture à l'environnement réel (physique ou social).

Les formes de consommation sentimentale basées sur des modes de séduction codés et extrêmement simplifiés sont l'objet central de ses sculptures. La connotation commerciale des personnages-mannequins fait allusion à la transformation de tout sentiment et émotion en marchandises-signes: plutôt que des objets, on consomme dorénavant des signes comme l'a suggéré Jean Baudrillard.

Du point de vue de l'histoire de l'art, la mise en scène des couples parodiait un sujet traditionnel (la représentation de l'amour, de la séduction) et, par là, amorçait un questionnement sur la nature de l'art (l'art savant, l'art populaire, l'art commercial) et sur sa fonction idéologique dans la sociétée 20 .

\section{Le simulacre comme signe du réel}

Les sculptures d'Hogenkamp étaient une réflexion sur la banalité et la banalisation des comportements entre personnes de sexe différent. La présentation matérielle de ces sculptures ne fonctionnant pas comme une clôture entre le public et l'objet d'art, leur base était réduite à une mince plaque qui épousait étroitement les parties des personnages reposant au sol; elles pouvaient être déposées n'importe où, de sorte qu'on pouvait tourner autour, les voir sous n'importe quel angle comme des objets en magasin.

Le contenu de l'œuvre était également très clair: elle représentait des rêves d'évasion véhiculés par les médias.

18 J. Richmond, "Anti-form and Anti-machines", The Gazette, 16 décembre 1967.

19 D. Cameron, article cité.

20 Cette critique de la société de consommation de signes par la représentation de l'amour romantique plastifié sera reprise et "travaillée" par Francine Larivée dans La Chambre nuptiale (1976) présentée au complexe commercial de la Place Desjardins (rue Sainte-Catherine est, Montréal). Cette artiste, pour bien souligner l'objectif de conscientisation sociale par l'art, adopta l'espace commercial comme lieu d'exposition de son environnement afin d'atteindre un public non spécialisé et d'interroger les liens de l'art actuel avec la conscientisation sociale. 


\section{Les couples}

Tout comme le couple des baigneurs, celui des danseurs est un ensemble figural en miroir. Le couple homme-femme est le modèle, le paradigme de la circulation entre réalités dissemblables ou opposées. Le rapport de séduction domine toujours mais il n'est pas aussi simple que la représentation le laisse entendre: la stratégie de l'artiste est de mettre en contradiction la simplicité des figures et la complexité du thème (les rapports de couple). La mise en scène des couples interrogeait, dans un esprit parodique, une situation vécue par la majorité des spectateurs.

L'artiste fait un constat adressé aux consommateurs des signes: "Ils [les couples sculptés] contrôlent la salle, écrivait le critique Normand Thériault . Et c'est le visiteur qui ne se sent pas chez lui, au lieu de ne voir que les sculptures (...). Et c'est là la force de l'exposition: elle n'en est pas une. Et loin de visiter des œuvres d'art, nous sommes dans un milieu vivant et notre réaction est personnelle et non pas centrée sur une réflexion d'analyse $(. . .)^{21}$."

L'apparente accessibilité matérielle de l'œuvre faisait écho à la supposée accessibilité des biens de consommation évoqués: s'étendre au soleil, danser, accéder à l'amour dans les lieux de loisirs.

Le parti pris plastique était de réduire l'objet (sculpture) à ses composants stricts et de réduire métaphoriquement le sujet évoqué (la relation de couple) à sa plus simple expression. Le contenu de l'œuvre était ainsi amplifié: le vide ou l'absence de communication répondait au stéréotype de la séduction instantanée.

Le thème du couple dansant est traité dans au moins deux œuvres de 1969-1970 (Discotheque Couple, Waltzing Couple): les rapports de séduction, transitoires et aléatoires, étaient mis en scène: il s'agissait moins de gestes instantanés comme chez un Duane Hanson par exemple qui figeait des situations prises sur le vif avec ses personnages hyper-réalistes mais isolés. Chez Hogenkamp, les personnages forment des mini-scènes narratives à la manière de courtes $\mathrm{BD}$. L'attention porte moins sur la nature des corps et leur matérialité que sur la situation qu'ils sont en train de vivre, le moment qu'ils traversent. Le cliché photographique sert ici de paradigme à la mise en scène tout en désignant le sens moral de lieu commun par les attitude évocatrices.

Les œuvres créaient donc une ambiance dans laquelle le public se [re]trouvait: quelques couples de danseurs, une table, quelques verres, une femme qui regarde la scène. Ces figures en polyester grandeur nature dansaient au son d'une musique de jazz et une projection sur écran géant montrait des séquences de vie quotidienne de gens d'âge moyen et d'enfants. D'autres se doraient au soleil. Un environnement

21 N. Thériault, "Voulez-vous danser mademoiselle?", La Presse, ler mars 1969. 
global était créé qui participait d'un engouement propre à cette fin des années soixante pour les mises en situation live $e^{22}$ : si les sculptures d'Hogenkamp n'appelaient pas une intervention directe de la part du public - hormis le fait de se promener entre des couples - du moins incitaient-elles à une participation imaginaire, virtuelle, puisque chaque élément de ces œuvres s'adressait à une expérience commune et se situait résolument dans un domaine proche de l'art populaire.

\section{Les rapports homme-femme comme contenu des sculptures}

En janvier 1966; la Galerie Innovation 66 du musée des Beaux-Arts de Montréal présenta une exposition des œuvres de Claire Hogenkamp ${ }^{23}$.

Gravures, collages, dessins et sculptures permettaient d'en saisir la trajectoire de 1963 à 1966 et de constater combien la représentation de stéréotypes (corps, attitudes, gestes) avait été au centre des préoccupations depuis le début des années soixante. Les gravures sur bois de la série 1963 s'intitulaient Feminine Mystique ${ }^{24}$, Miss Universe, Haute Couture, The Apartment, The Lovers, Mother des collages de 1963-1965 s'intitulaient The Bride, The Hero. Quant aux sculptures, leurs titres et leurs thèmes renvoyaient aux codes tant féminins que masculins des figures médiatiques: Golden Girl au maquillage naturaliste et à l'allure "sexy", Je les fume parce que je les aime (femme assise sur un vrai banc, portant un vrai chemisier) ou encore The Man for the Job, déconstruisaient des stratégies publicitaires qui faisaient croire à un comportement naturel. La charge était particulièrement concentrée sur la représentation de la féminité dans les médias, et sur la portée sociale de cette féminité.

\section{Au coeur de l'émotion par la représentation de comportements banalisés}

La critique des rapports homme-femme était nouvelle dans le champ des arts visuels au Québec. Mais encore plus novateur était de la mener avec les moyens mêmes qui assuraient l'émergence sur la scène sociale des attitudes véhiculées par les médias. Cette fonction critique de l'art prolongeait la tendance de l'art à contenu social, qu'a rappelée l'exposition Peintres juifs et modernité 1930-194525.

22 Tels les happenings, la murale de Mousseau qui éclairait le hall d'entrée de l'édifice de l'Hydro-Québec, boulevard René-Lévesque, Montréal.

23 En même temps que celles de Polken.

24 B. Friedan avait publié The Feminine Mystique en 1963.

25 Présentée au Centre Saidye Bronfman en 1987. La conservatrice était Esther Trépanier qui rédigea également le catalogue Peintres juifs et modernité 1930-1945, Centre Saidye Bronfman, Montréal, 1987. 
Claire Hogenkamp était reliée au monde artistique anglophone où la tradition de l'art à visée sociale était vivante. À Montréal depuis 1947, anglophone, elle était sensible à cette tradition.

À cette époque, la Révolution tranquille au Québec achevait sa première phase mais les transformations dans le champ de l'art s'étaient amorcées avec les Automatistes et le Refus Global depuis deux décennies. Quand Hogenkamp exposa ses sculptures, l'euphorie de la Révolution tranquille était déjà tempérée par des remises en question sur le plan social et culturel mais aussi sur le plan des relations personnelles et interpersonnelles: un esprit nouveau se développait, bien différent de celui qu'avaient imaginé, dans les années cinquante et la première moitié des années soixante, les artistes post-automatistes de la Révolution tranquille. Il est même arrivé qu'un critique d'art anglophone, devant la tendance critique des artistes québécois, l'attribue à un contexte socio-culturel et géographique singulier: "Perhaps artistic nihilism is a logical product of the politics of this province; perhaps it may simply be a reaction to the wholesale adoption of the values and toys from south of the border ${ }^{26}$." La dérision, l'ironie qui, pour ce critique, caractérisaient les œuvres d'Hogenkamp étaient menacées, pour un autre (Yves Robillard), par l'esthétisation de la consommation ou du commerce.

Quoi qu'il en soit, la question était clairement posée: à travers l'examen des comportements entre homme et femme, elle questionnait la fonction critique de l'art, non en tant qu'illustration de quelque cause sociale mais dans l'organisation de ses composants formels mêmes.

Sa critique sociale des comportements stéréotypés homme-femme s'est ainsi faite à même une figuration dépouillée des subtilités visuelles propres aux œuvres des Plasticiens (art savant), représentants officiels de l'art au Québec après 1965.

Son entreprise critique, dans sa forme figurative, fut unique parmi le groupe de jeunes sculpteurs dont Hogenkamp faisait alors partie à Montréal. Serge Cournoyer avec sa plante arrosée mécaniquement, relevait lui aussi la mécanisation des rapports humains et les rapports problématiques entre art et technologie. Ces sculpteurs étaient en même temps fascinés par les objets de consommation (Pierre Ayot).

Si Hogenkamp fut pratiquement la seule femme sculpteur à formaliser des rapports interpersonnels et subjectifs, elle le fit en s'appropriant des formes populaires et commerciales de représentation déjà existantes et accessibles et désigna de manière paradoxale le sujet en dévoilant le processus d'aliénation et de banalisation dont il est l'objet (images publicitaires...).

26 J. Richmond, "Anti-form and Anti-Machines", The Gazette, 16 décembre 1967. 
Le fait de s'être elle-même représentée dans une de ses œuvres ( $S$ un Worshippers) montre bien la nature de son regard sur les personnages sculptés: l'art est vécu comme autoportrait, autoreprésentation et, par là, il établit un lien organique avec le contexte où il est produit et se produit. L'humour, l'ironie, mais non la dérision imprègnent les œuvres de Hogenkamp.

L'artiste ne s'est pas soustraite à son propre regard et, partant, n'a pas porté de jugement moralisateur ni sur les personnages représentés ni sur les thèmes traités. Elle a néanmoins désigné un système de société, le sien propre, comme lieu premier de fabrication des signes-marchandises matériels et émotionnels.

Elle aura ainsi proposé une relation différente entre art et société dans cette perspective précise où l'empathie entre l'œuvre, son public et elle-même (donc sur une relation entre les choses) aura su fonctionner.

\author{
Rose-Marie ARBOUR \\ Département d'histoire de l'art \\ Université du Québec à Montréal
}

\title{
Résumé
}

Au départ l'auteure souligne que les travaux de la sculpteure qu'elle étudie font partie du courant figuratif ayant contesté, au cours des années soixante, le formalisme en arts visuels. En commentant des œuvres présentées à l'exposition "People in the Park", l'auteure montre que Claire Hogenkamp a pratiqué une satire marquée, mais indulgente (non culpabilisante) de la féminité et de la masculinité construites essentiellement sur des rapports de séduction hypercodés suggérés par l'imagerie publicitaire et la photographie commerciale. Elle précise que le contenu de ces œuvres, par leur effet de distanciation du réel, montre l'évidence du règne du faux, particulièrement dans le cas de la féminité construite par les médias commerciaux. Finalement, l'auteure démontre que cette recherche d'Hogenkamp constitue une critique sociale forte des comportements homme-femme stéréotypés, ce qui du coup révèle la fonction idéologique de l'art, c'est-à-dire sa nécessaire portée critique.

\section{Summary}

The author begins by situating Claire Hogenkamp's sculptures within the 1969s figurative current which took a stand against formalism in the visual arts. Commenting certain works presented in the exhibition "People in the Park", the author shows how Hogenkamp's work can be defined in terms of a marked, but indulgent - void of any guilt-induced elements - satire of the notions of femininity and masculinity, based essentially on the hypercoded rapports of 
seduction suggested by both advertising imagery and commercial photography. The author argues that the content of these sculptures - with their effect of distanciation from what is real - bears testimony to the reign of simulacrum, particularly in the case of commercial media-constructed femininity. Finally, the author demonstrates how Hogenkamp's research constitutes a strong social critique of stereotypical male/female behaviour patterns, which in turn, points to the ideological function of art, more precisely to its necessary critical discourse. 